\title{
RECURRENCE OF THE BLUE WING ENHANCEMENTS IN THE HIGH-IONIZATION LINES OF SDSS 1004+4112A
}

\author{
P. Gómez-Álvarez, ${ }^{1}$ E. Mediavilla, ${ }^{1}$ J. A. Muñoz, ${ }^{2}$ S. Arribas, ${ }^{1,3,4,5}$ S. F. Sánchez, ${ }^{6,7,8}$ \\ A. Oscoz, ${ }^{1}$ F. Prada, ${ }^{6}$ AND M. Serra-Ricart \\ Received 2005 April 4; accepted 2006 May 23; published 2006 June 27
}

\begin{abstract}
We present integral field spectroscopic observations of the quadruple-lensed QSO SDSS 1004+4112 taken with the fiber system INTEGRAL at the William Herschel Telescope on 2004 January 19. In 2003 May, a blueward enhancement in the high-ionization lines of SDSS 1004+4112A was detected, and then it faded. Our observations are the first to note a second event of similar characteristics less than 1 year after. Although initially attributed to microlensing, the resemblance among the spectra of both events and the absence of microlensinginduced changes in the continuum of component $\mathrm{A}$ are puzzling. The lack of a convincing explanation under the microlensing or intrinsic variability hypotheses makes the observed enhancements particularly relevant, calling for close monitoring of this object.
\end{abstract}

Subject headings: gravitational lensing — quasars: emission lines — quasars: individual (SDSS 1004+4112)

\section{INTRODUCTION}

The large-separation (14."62), quadruple gravitationally lensed quasar was first identified by Inada et al. (2003, hereafter I03) in the Sloan Digital Sky Survey (SDSS). The derived redshift is $z=1.732$, and the lensing complex consists of a cluster of galaxies at $z=0.68$.

The components of a multiple-lensed system can show uncorrelated variability due to the gravitational (de)magnification produced by compact deflectors aligned with the source. This phenomenon, microlensing (Chang \& Refsdal 1979, 1984), is routinely observed in the continuum emission of multiple-imaged QSOs, and it is considered a powerful tool for mapping the QSO continuum source (Yonehara et al. 1999).

According to our current understanding of the structure of active galactic nuclei (AGNs), the continuum source of a QSO is surrounded by a wider region, where the broad emission lines (BELs) originate. Early versions of the standard model of AGNs assumed a too large size for the broad-line region (BLR; $\sim 1 \mathrm{pc}$ ) to be microlensed by solar-mass objects (Nemiroff 1988; Schneider \& Wambsganss 1990). However, these early estimates were reduced by 2 orders of magnitude by the new measurements of the BLR size based on the reverberation method (Wandel et al. 1999; Kaspi et al. 2000). Considering the new size estimates, a variety of theoretical models for the kinematic structure of the BLR (Popović et al. 2001; Abajas et al. 2002; Lewis \& Ibata 2004) have shown that microlensing by stellar-sized objects can produce significant amplifications of the BEL of multiple-imaged QSOs, especially in the highionization lines.

\footnotetext{
${ }^{1}$ Instituto de Astrofísica de Canarias, Vía Láctea s/n, E-38200 La Laguna, Tenerife, Spain.

${ }^{2}$ Departamento de Astronomía y Astrofísica, Universidad de Valencia, E46100 Burjassot, Valencia, Spain.

${ }^{3}$ Space Telescope Science Institute, 3700 San Martin Drive, Baltimore, MD 21218.

${ }^{4}$ Affiliated with the Space Telescope Division of the European Space Agency, ESTEC, Noordwijk, Holland.

${ }^{5}$ Consejo Superior de Investigaciones Científicas (CSIC), C/ Serrano 117, E-28006 Madrid, Spain.

${ }^{6}$ Instituto de Astrofísica de Andalucia, CSIC, E-18008 Granada, Spain.

${ }^{7}$ Astrophysikalisches Institut Postdam, An der Sternwarte 16, 14482 Potsdam, Germany.

${ }^{8}$ Calar Alto Observatory, Centro Astronómico Hispano Aleman, Apartado 511, E-04044 Almería, Spain.
}

Richards et al. (2004b, hereafter R04b) found excess in the blue wings of several high-ionization lines of SDSS 1004+ 4112A, the highest magnified lensed image, relative to the same lines in the other images. The excess persisted for at least 28 days and then faded. No such enhancements in the blue wings were seen in the low-ionization lines. These results are in agreement with current ideas about the stratification of the BLR according to the ionization level (Peterson \& Wandel 2000) and suggest the possibility of scanning the BLR with high spatial resolution. There is no evidence of continuum microlensing in the SDSS 1004+4112 event (however, see Ota et al. 2006). This lack of correlation can be explained for a particular event (Lewis \& Ibata 2004), but the apparent absence of continuum amplification in all the reported cases of BEL microlensing (R04b; Chartas et al. 2002, 2004; Dai et al. 2003) is intriguing.

In this article we present new spectroscopic measurements of SDSS $1004+4112$ that show a recurrence of the blueward magnification of the high-ionization line wings of image A.

\section{OBSERVATIONS AND DATA REDUCTION}

SDSS $1004+4112$ was observed on 2004 January 19 with the fiber system INTEGRAL (Arribas et al. 1998), at the $4.2 \mathrm{~m}$ William Herschel Telescope (WHT; Roque de los Muchachos Observatory, La Palma, Spain). Two sets of three $1800 \mathrm{~s}$ exposures were taken corresponding to two pointings separated by a few arcseconds. We use the standard fiber bundle 3 (SB3) that consists of 115 objects +20 sky fibers, each one subtending 2".70 in diameter and covering a sky area of 33"6 × 29".4. A $300 \mathrm{~mm}^{-1}$ diffraction grating was used, giving a spectral coverage of $\sim 6000 \AA$ centered at $5700 \AA$ and a spectral resolution of $19 \AA$, with a spectral dispersion of $6.2 \AA$ pixel $^{-1}$. The observations were performed under medium seeing $\left(1 " .5-2^{\prime \prime}\right)$ conditions. The data reduction procedures included cosmic-ray rejection, aperture tracing, spectra extraction, wavelength calibration, throughput correction, sky subtraction, atmospheric extinction correction, and flux calibration. Since the vast majority of the flux is concentrated in one or two fibers, the spectrum has been obtained directly by combining their fluxes.

The absolute flux calibration must be treated with caution since the calibration star position within the fiber face could affect the amount of flux lost in the interfiber regions. The 
TABLE 1

SUMmaRY OF SDSS $1004+4112$ OBSERVATIONS

\begin{tabular}{|c|c|c|c|c|}
\hline Date & $\Delta T^{\mathrm{a}}$ & Telescope & Ref. & Components \\
\hline 2003 May $31 \ldots .$. & 0 & Keck & $\mathrm{R} 04 \mathrm{~b}$ & $\mathrm{ABCD}$ \\
\hline 2003 Nov $21 \ldots$ & 174 & ARC & R04b & $\mathrm{AB}$ \\
\hline 2003 Nov $30 \ldots . .$. & 183 & ARC & $\mathrm{R} 04 \mathrm{~b}$ & $\mathrm{AB}$ \\
\hline 2003 Dec $1 \ldots \ldots$. & 184 & ARC & $\mathrm{R} 04 \mathrm{~b}$ & $\mathrm{AB}$ \\
\hline 2003 Dec $22 \ldots \ldots$ & 205 & $\mathrm{ARC}$ & $\mathrm{R} 04 \mathrm{~b}$ & $\mathrm{AB}$ \\
\hline 2004 Jan $19 \ldots \ldots$ & 233 & WHT & $\ldots$ & $\mathrm{ABCD}$ \\
\hline 2004 Mar 26 ...... & 300 & $\mathrm{ARC}$ & $\mathrm{R} 04 \mathrm{a}^{\mathrm{b}}$ & $\mathrm{AB}$ \\
\hline
\end{tabular}

${ }^{a}$ Elapsed days from first observation

${ }^{\mathrm{b}}$ Richards et al. (2004a).

goodness of our relative photometry is shown by the comparison with the photometry of I03 (Figs. 3 and 4 below).

Apart from WHT observation, we have use data from the Keck and Astrophysical Research Consortium (ARC) telescopes (see Table 1), which have been kindly provided by G. T. Richards.

\section{RESULTS}

\subsection{Comparison of A, B, and C Spectra}

Inspection of Figure 1 reveals that (1) component A shows enhancements relative to components $\mathrm{B}$ and $\mathrm{C}$ in the blue wings of the high-ionization emission lines ( $\mathrm{Si}$ IV/O IV], C IV, He II, Al III, and the UV48 Fe III complex) similar to those found by R04b; (2) the A and B continua match very well in all of the observed range, although the $\mathrm{C}$ continuum exhibits an excess beyond $2000 \AA$; (3) the low-ionization lines, C III] and Mg II, match reasonably well, with the same normalization factor as the continua; and (4) the EWs of the low-ionization lines $\mathrm{C}$ III] and $\mathrm{Mg}$ II are larger in $\mathrm{C}$ than in $\mathrm{A}$, and the $\mathrm{EW}$ of the $\mathrm{C}$ IV line is larger in $\mathrm{C}$ than in $\mathrm{B}$.

\subsection{Comparison with Spectra of Other Epochs}

Seven spectroscopic observations were made between 2003 May 31 and 2004 March 26 (see Table 1). The differences between the WHT spectra corresponding to components A and $\mathrm{B}$ are remarkably similar to those reported by R04b and Oguri et al. (2004) from Keck observations taken on 2003 May 31. A direct comparison, not shown here (see Figs. 3 and 4 below), among our spectra and the Keck 2003 data shows a very good global agreement for components A, B, and C. In the case of component $\mathrm{A}$, some differences are found in $\mathrm{C}$ IV (the $\mathrm{EW}$ is larger in the WHT spectrum) and in He II (the blueward asymmetry is stronger in the Keck spectrum).

A direct comparison (not shown here) of the A and B ARC spectra based on the normalization of the continua shows that during the epoch 2003 November 21-December 22, the blue excess disappears in the line of highest ionization (He II) and severely declines in $\mathrm{C} \mathrm{IV}$, but it is still present in the lower ionization Al III and Fe III UV48 lines. The WHT observations show that the blue enhancements in component $A$ again reached (on 2004 January 19) a level comparable to that of the Keck observations (2003 May 31). In Figure 2 we see that on 2004 March 26, the system shows features similar to the WHT and Keck observations.

\subsection{B - A and C - A Magnitude Difference Curves}

We estimate the flux ratio independently for the continuum and the emission lines. For the high-ionization lines, we have integrated only the blue side to enhance the asymmetries. To obtain the emission-line flux, we subtract a linear interpolation

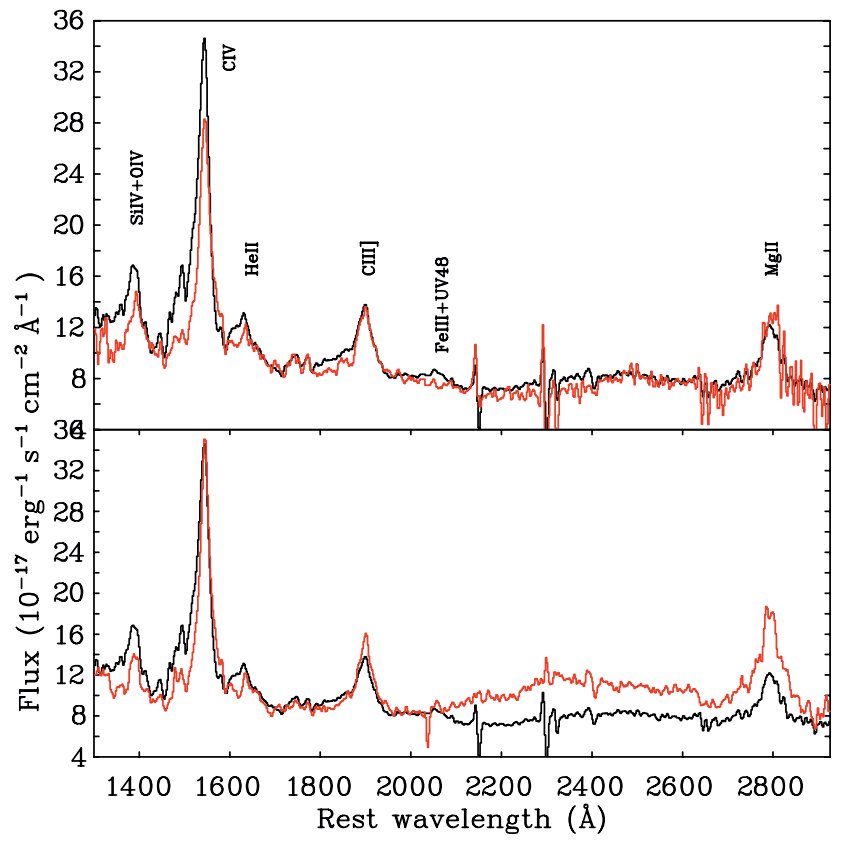

FIG. 1.-Comparison between spectra of components A (black line in both panels), B (upper panel), and C (lower panel) for SDSS 1004+4112 at the WHT. Spectra B and C have been scaled to normalize their continuum to that of $\mathrm{A}$ in the 1700-1800 $\AA$ wavelength range.

of the continuum from the regions adjacent to the lines. We then computed the continuum flux over intervals of $200 \AA$. The resulting magnitude differences, $\Delta m_{B A}=m_{B}-m_{A}$ and $\Delta m_{C A}=m_{C}-m_{A}$, for both the continuum and the emission lines are presented in Figures 3 and 4.

In Figure 3 we present the WHT and Keck data. The agreement between both observations is quite remarkable. The small offset ( $0.05 \mathrm{mag}$ ) between the Keck and WHT continuum difference curves strongly constrains the microlensing-induced continuum variability between both epochs (2003 May 31 and 2004 January 19). There is also good agreement (better than 0.05 mag with respect to the WHT continuum curve) with the photometric data by I03. This is also reasonable in terms of the intrinsic variability if we take into account that the yearly

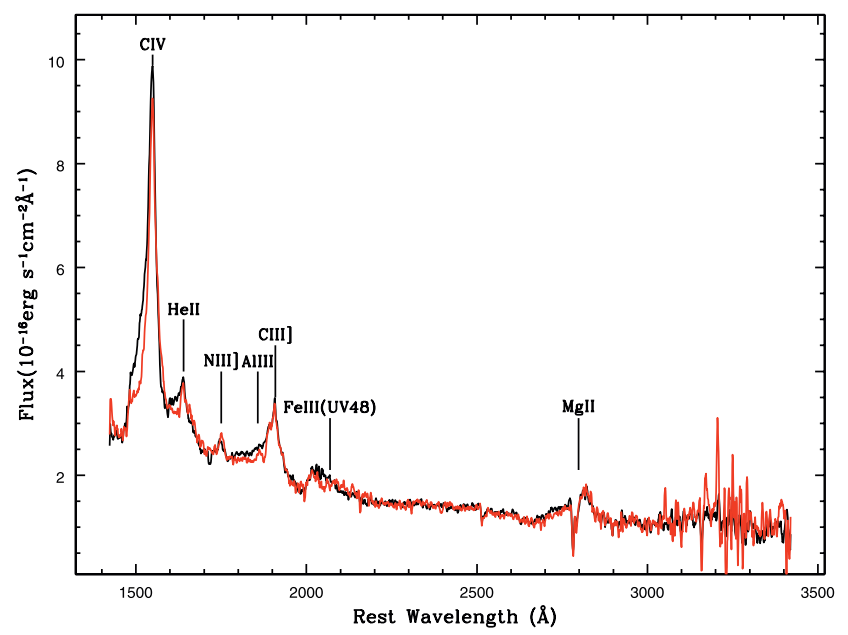

FIG. 2.-SDSS 1004+4112A/B spectra taken on 26 March 2004 at the ARC telescope. 


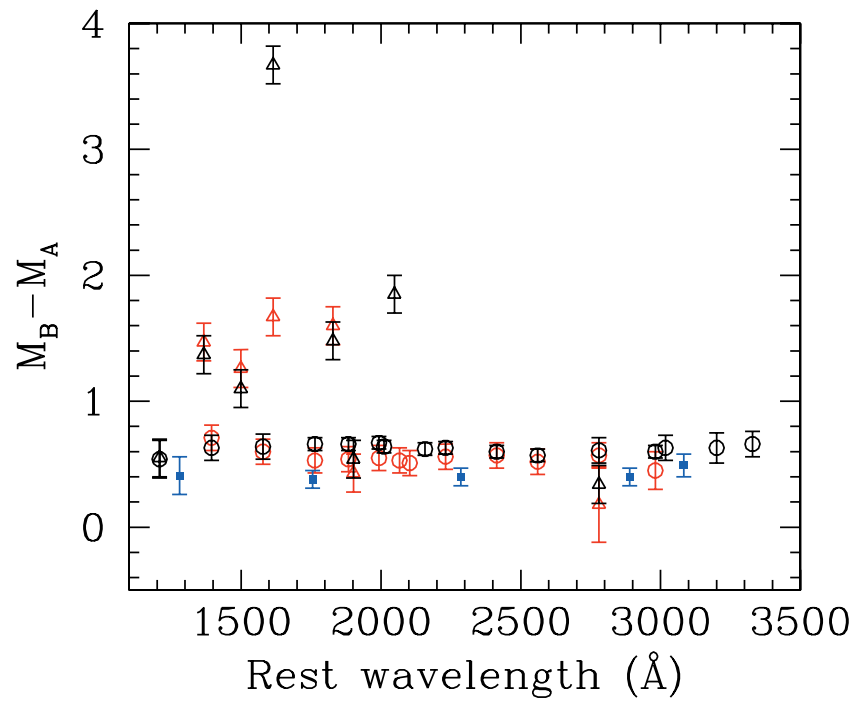

Fig. 3.-B - A magnitude difference curve. Triangles: Emission lines. Circles: Continua. Blue squares: Photometric data from I03. Red symbols: WHT-INTEGRAL. Black symbols: Keck-LRIS.

intrinsic variation of this object could be about $0.2 \mathrm{mag}$ (according to the preliminary results of the photometric monitoring that our group is performing) and that the predicted time delay between components $\mathrm{A}$ and $\mathrm{B}$ is about 1 month.

The B - A differences for the low-ionization lines (C III], $\mathrm{Mg}$ II) match (within errors) the continuum difference curve (there is some marginal evidence of a negative offset for Mg II). This result does not support the existence of microlensing in the continuum. The blue sides of the high-ionization lines are above the continuum, with an offset of about $1 \mathrm{mag}$ for Si IV, C IV, $\mathrm{Al}$ III, and the UV48 Fe III complex. The blue side of the He II line shows a larger offset of about 3 mag respect to the continuum. Finally, there is no evidence of the wavelength dependence of the continuum difference curves that have been invoked in several cases (see Wucknitz et al. 2003 and Motta et al. 2002) to justify chromatic microlensing. This also excludes the possibility of differential dust extinction.

The $\mathrm{C}-\mathrm{A}$ difference magnitude curves are shown in Figure 4. The agreement between the WHT difference curve for the continuum and the photometric data by $\mathrm{I} 03$ is also remarkably good and does not support the existence of noticeable microlensing variability during the period between both observations. The Keck spectrum needed an offset of 0.8 mag to be matched to that of the I03 data. In Figure 4 the high-ionization lines appear above the continuum, but the low-ionization lines do not match the continuum curve, which is $\sim 0.45 \mathrm{mag}$ above. An offset between the line and continuum quotients of two components of a gravitational lens has been interpreted as evidence of microlensing (see Mediavilla et al. 2005 and Motta et al. 2002). If we assume that this hypothesis can be applied to the low-ionization BEL and if we take into account that, for the same reason, microlensing in component $\mathrm{A}$ is unlikely, we should accept that the continuum of component $\mathrm{C}$ is demagnified by microlensing. On the other hand, the continuum difference curve is not flat but shows a relative decrease in $\mathrm{C}$ with respect to $\mathrm{A}$ for shorter wavelengths (the color difference between components $\mathrm{A}$ and $\mathrm{C}$ has already been reported during the identification of the system as a multiple-imaged QSO of large separation; see I03). This can be easily interpreted as the dust extinction of component $\mathrm{C}$ (see, e.g., Falco et al. 1999 and Muñoz et al. 2004). An interesting

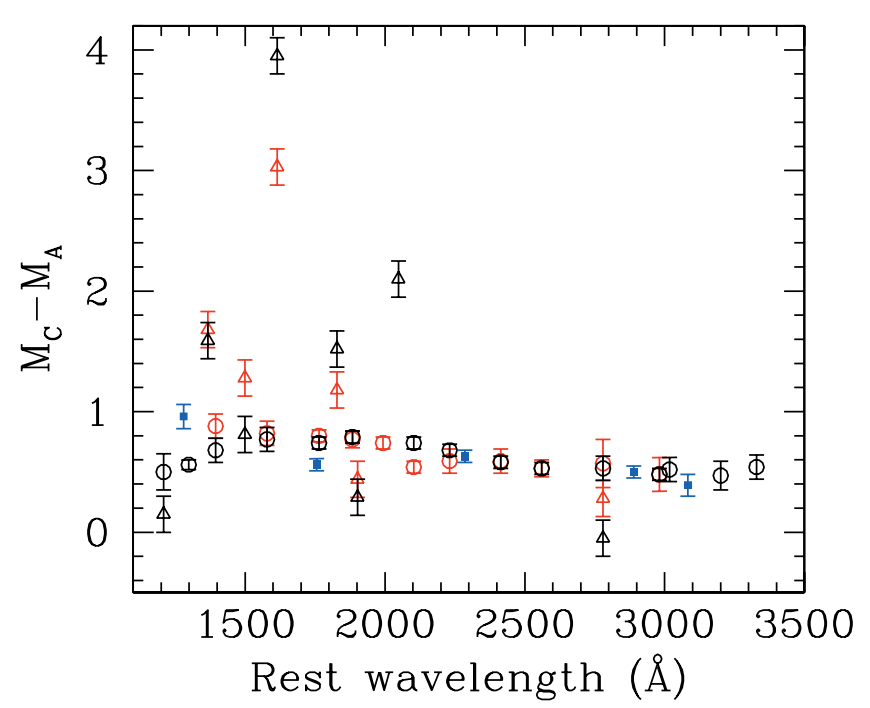

FIG. 4.- C - A magnitude difference curve. Triangles: Emission lines. Circles: Continua. Blue squares: Photometric data from I03. Red symbols: WHT-INTEGRAL. Black symbols: Keck-LRIS.

alternative possibility to explain the color differences around $2100 \AA$ and longer (see Fig. 1) is that the UV Fe II emission is microlensed (magnified) in the $\mathrm{C}$ image and not in the others. The empirical iron emission template given by Vestergaard \& Wilkes (2001) matches qualitatively the observed variation with wavelength. Both possibilities can be discriminated with future observations since microlensing in the iron lines would show time variability while extinction would not.

\section{B - A VARIABILITY FOR THE He II AND C III] EMISSION LINES}

In Figure 5 we present the $\mathrm{B}-\mathrm{A}$ values for the $\mathrm{C}$ III] and $\mathrm{He}$ II (blue side) emission lines corresponding to the seven epochs with spectra available (see § 3.2). The B - A value for the low-ionization $\mathrm{C}$ III] line is almost constant (matching the $\mathrm{B}-\mathrm{A}$ value for the continuum; see previous section), whereas the He II emission line exhibits strong variability from a maximum during the first epoch, a posterior decay, and a final rise. It is very noticeable that the minimum of $\mathrm{B}-\mathrm{A}$ for $\mathrm{He}$ II coincides with the baseline defined by $\mathrm{C}$ III].

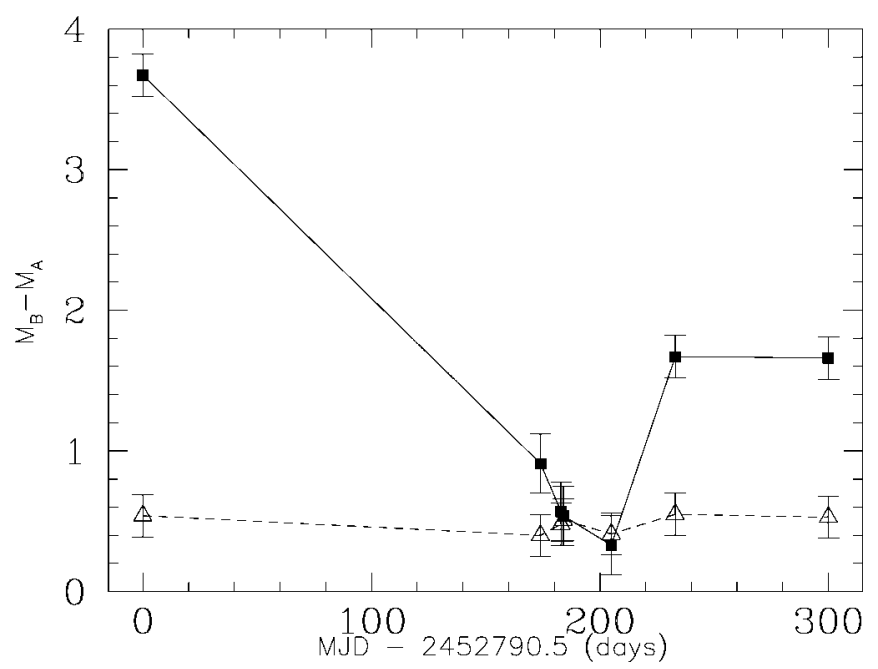

FIG. 5.-Magnitude differences between components A and B for C III] (triangles) and $\mathrm{He}$ II lines (squares) 


\section{DISCUSSION AND CONCLUSIONS}

The interpretation of the apparent recurrence of the blueward enhancement of the high-ionization lines is challenging. If the enhancements were produced by intrinsic variability, they should appear with some delay in all of the components. However, observations do not reveal changes in component $\mathrm{B}$ that could be related to the blue wing asymmetries detected in component A. On the other hand, the lack of a continuum variability that might induce the change in the emission lines also argues against this hypothesis.

Alternatively, the rising and fading of the blue wing enhancements can be explained by the relative displacement of a caustic network with respect to the BLR. However, the displacement of the caustic across a kinematically structured BLR should induce velocity changes in the high-ionization lines (I03) rather than recurrences like the one observed. Another inconvenience of the microlensing hypothesis is the very short timescale for emission-line variability. According to the observations, the He II blue wing excess was not present in the ARC 2003 December 22 spectrum but appears in the WHT spectrum taken on 2004 January 19 (see Fig. 5), implying a rise time of less than 1 month, which reduces by a factor of $\sim 6$ the upper limit inferred by R04b for the size of the amplified region under the hypothesis of microlensing. R04b estimated that the impact of microlensing in the A continuum was no more than $20 \%$ during the time spanned by their observations. Our comparison (see previous section) among the I03, Keck, and WHT data indicates no traces of B - A continuum variability during the period between 2003 May 2 and 2004 January 19. On one hand, this implies that the continuum microlensing timescale (if any) should be much larger than the one associated with high-ionization line variability. On the other hand, (1) the agreement among the $\mathrm{B}-\mathrm{A}$ data corresponding to the lowionization lines and the continuum and (2) the apparent absence of microlensing chromaticity do not support this hypothesis. Thus, it seems that the process causing the variability of the high-ionization lines does not affect the continuum. According to Lewis \& Ibata (2004) and R04b, microlensing by a caustic network can cause uncorrelated variability between the highionization lines and the continuum. However, it is difficult to explain the absence of significant continuum microlensing $[\Delta(\mathrm{B}-\mathrm{A})<0.05 \mathrm{mag}]$ when the high-ionization lines have undergone a complete cycle that, in the case of the blue wing of He II, implies $\Delta(\mathrm{B}-\mathrm{A})$ differences of several magnitudes.

The decoupling between continuum and emission lines can be explained by supposing that the emission-line region is formed by clouds of ionized gas moving at high velocity and/ or considering a special geometry (biconical) for the BLR. This would favor the appearance of asymmetrical features and might explain the uncorrelated variability between the emission lines and the continuum. Even the possibility of microlensed transient events as supernovae or shocks deserves consideration. We will explore these possibilities in future work.

We are grateful to Gordon T. Richards for providing previous observations and useful comments. We also wish to acknowledge the referee, Ian Browne, for valuable comments and ideas. This work was supported by the European Community's Sixth Framework Marie Curie Research Training Network Programme, contract MRTN-CT-2004-505183 “ANGLES," and by the Ministerio de Educación y Ciencia of Spain with the grants AYA2004-08243-C03-01 and AYA2004-08243-C03-03.

\section{REFERENCES}

Abajas, C., Mediavilla, E., Muñoz, J. A., Popović, L. Č., \& Oscoz, A. 2002, ApJ, 576, 640

Arribas, S., et al. 1998, Proc. SPIE, 3355, 821

Chang, K., \& Refsdal, S. 1979, Nature, 282, 561 1984, A\&A, 132, 168

Chartas, G., Agol, E., Eracleous, M., Garmire, G., Bautz, M. W., \& Morgan, N. D. 2002, ApJ, 568, 509

Chartas, G., Eracleous, M., Agol, E., \& Gallagher, S. C. 2004, ApJ, 606, 78

Dai, X., Chartas, G., Agol, E., Bautz, M. W., \& Garmire, G. P. 2003, ApJ, 589,100

Falco, E. E., et al. 1999, ApJ, 523, 617

Inada, N., et al. 2003, Nature, 426, 810 (I03)

Kaspi, S., Smith, P. S., Netzer, H., Maoz, D., Jannuzi, B. T., \& Giveon, U. 2000, ApJ, 533, 631

Lewis, G. F., \& Ibata, R. A. 2004, MNRAS, 348, 24

Mediavilla, E., Muñoz, J. A., Kochanek, C. S., Falco, E. E., Arribas, S., \& Motta, V. 2005, ApJ, 619, 749
Motta, V., et al. 2002, ApJ, 574, 719

Muñoz, J. A., Falco, E. E., Kochanek, C. S., McLeod, B. A., \& Mediavilla, E. 2004, ApJ, 605, 614

Nemiroff, R. J. 1988, ApJ, 335, 593

Oguri, M., et al. 2004, ApJ, 605, 78

Ota, N., et al. 2006, ApJ, in press (astro-ph/0601700)

Peterson, B. M., \& Wandel, A. 2000, ApJ, 540, L13

Popović, L. Č., Mediavilla, E. G., \& Muñoz, J. A. 2001, A\&A, 378, 295

Richards, G., Johnston, D., \& Hennawi, J. 2004a, IAU Circ., 8325, 2

Richards, G. T., et al. 2004b, ApJ, 610, 679 (R04b)

Schneider, P., \& Wambsganss, J. 1990, A\&A, 237, 42

Vestergaard, M., \& Wilkes, B. J. 2001, ApJS, 134, 1

Wandel, A., Peterson, B. M., \& Malkan, M. A. 1999, ApJ, 526, 579

Wucknitz, O., Wisotzki, L., Lopez, S., \& Gregg, M. D. 2003, A\&A, 405, 445

Yonehara, A., Mineshige, S., Fukue, J., Umemura, M., \& Turner, E. L. 1999, A\&A, 343, 41 